It is now universally recognized that every city of any considerable size requires an institution for the care and detention of the large number of persons temporarily or permanently incapacitated from a mental standpoint-in other words, the psychopathic hospital is more and more seen as a necessity. Such hospitals are already in beneficial operation, but their usefulness could be largely increased by making them more free of access. Patients should be admitted in the same manner as those admitted to other hospitals and freely discharged as well, except in the case of patients who are in a condition making it unsafe or injurious to themselves or others to be at large.

A considerable number even of those who, strictly speaking, are insane, would offer no objection to their stay in the hospital; while the great majority of those suffering from hysteria or severe neurasthenia, victims of drink or drugs, or patients with toxic delirium or cerebral hemorrhage, tumor or trauma could go to the psychopathic hospital, which is by far the most appropriate place for their treatment, precisely as to any other hospital. If any of either class needed detention against their will, then and then only would occasion arise for invoking the authority of the law, and for detention by order of court. All others might be free to go and come according to their own will. No so-called ban or stigma or verdict of insanity would unnecessarily be placed on any, and the court, with the aid of medical advice, would be able to discriminate between such cases and those of psychoses actually requiring the intervention of judge or jury. Furthermore, the so-called ban or stigma of insanity would be largely removed by the very policy here proposed.

In this connection, it is pertinent to speak of the meaning of the word "insanity." This word has never been defined satisfactorily from either the legal or the medical standpoint. It is as indefinite when applied to the mind as is the word "sick" when applied to the body. Just as there are all possible degrees of sickness, so there are infinite gradations of so-called insanity. Some bodily sickness is so severe that it confines a patient to bed; in other cases, the patient is up and about; patients may be seriously and incurably sick and yet walk the streets and transact business. In the same way the term "insanity" is applied to innumerable conditions. In some cases, it means total loss of mind. In others, a great degree of mental clearness remains. Some patients need close confinement; others can have entire freedom; still others, though decidedly insane, are bright and capable in certain directions; some patients are only temporarily or periodically affected. Insanity is a word without any uniform significance. It would be well if it could be omitted from scientific writings. The term "psychosis" is applicable to all forms of mental disorder or derangement, and carries with it no offense.

The psychopathic or detention hospital as now constituted is in all cases under jurisdiction of the county or city court, which determines the status of the inmates from time to time and warrants their commitment, if necessary, to the state institutions. In some instances, the court sits and keeps its record in the hospital building, provision being made for this in its construction. Such is the arrangement now existing at the psychopathic hospital in Chicago for Cook County, and it is believed that if such arrangements were made at the psychopathic hospitals of New York, Boston and other large cities, the public, especially the "psy- chopathic" public, would be the gainer. Such provision could readily be made in all cases, and the machinery of the court could be so arranged that at regular and stated intervals each inmate of the psychopathic hospital could be interrogated by the clerk or other independent agent of the court as to his or her desire to remain under treatment or to leave the hospital. A 11 who wished to leave, whose condition required legal control, could readily be dealt with in the same manner as is now customary; the difference would be that under this provision a very much smaller number of cases would require commitment or other interference from the law or court.

\section{TREATMENT OF NONTUBERCULOUS LUNG ABSCESS WITH PNEUMOTHORAX}

\author{
REPORT OF TEN CASES * \\ W. D. TEWKSBURY, M.D.
}

Superintendent, Tuberculosis Hospital; Physician in Charge, Tuberculosis Dispensary

WASHINGTON, D. C.

In a paper published last year, I reported ${ }^{1}$ two cases of acute pulmonary abscess treated with artificial pneumothorax, stating that so far as I knew, this was an original method of treatment for acute abscesses. The acute abscess is not a rare condition, since, after the publication of my report, I received a large number of letters from physicians who had abscess cases under treatment. In Washington alone during the past year I have seen fifteen cases following operations on the nose or throat.

In this report, I discuss only the lung abscess that follows an operation on the nose or throat. It may be well to state briefly the etiology, symptoms, etc., common to these cases.

The infection is of the usual mixed type, with the staphylococcus and streptococcus predominating. There are two theories as to the mode of infection: 1. The infecting organisms are carried into the lung by the direct aspiration of infected blood, due to a partial paralysis of the epiglottis while the patient is under the anesthetic. 2. The infecting organisms are carried into the lung from the field of operation by means of an infected clot, with a resulting septic infarct. The symptoms appear from four to ten days after operation, and begin with a rise in temperature, dry cough, pain in the chest on the affected side, and profuse sweating. Blood examination shows a leukocytosis, and physical examination reveals dulness with diminished breathing over the abscessed area. From one to two weeks after onset of the symptoms, the abscess ruptures, and a large amount of foul pus is coughed up. The prognosis under medical treatment is poor. In rare instances, when the abscess is small, healing takes place spontaneously. I have seen this occur in one instance. Forchheimer ${ }^{2}$ quotes Kiss̄ling's report of 133 cases of lung abscess and lung gangrene treated medically, with less than 10 per cent. resulting in recovery, and 64 per cent. in death. I myself have seen seven cases treated medically without pneumothorax. Of these, four patients died, two are improved, and one made an apparent recovery.

* Read before the George Washington Medical Society, Dec. 15, 1917
1. Tewksbury, W. D.: Acute Pulmonary Abscess Treated with Artificial Pneumothorax, The Journal A. M. A., March 10, 1917, p. 770. 2. Forchbeimer, Frederick: Therapeusis of Internal Diseases, New York, Daniel Appleton \& Co., 1913, 3, 441. 
The first eight cases reported in this series were acute, being of less than six weeks' duration.

\section{REPORT OF CASES}

CASE 1.-Mr. H. G. P., aged 32, seen in consultation with Drs. Reginald Walker, Charles White and Sterling Ruffin, was operated on, June 17, 1916. He developed an abscess of the right lung, June 26 , and was becoming progressively worse until August 12, about six weeks after onset of the symptoms, when pneumothorax treatment was started. The lung was gradually compressed by a small and frequent injection of air into the pleural sac, and later, large and less frequent injections were used. Ten treatments were given from August 12 to October 15, on which date the patient was discharged as cured. Roentgenograms taken several months after the discharge showed the lung as normal, with practically no evidence of the abscess scar. The patient is in perfect health now, iourteen months after treatment.

CASE 2.-Mrs. O. B., aged 27 , seen in consultation with Dr. Charles W". Richardson, was operated on, Sept. 9, 1916. September 16, an abscess of the left lung developed. The patient was extremely septic, becoming rapidly worse. October 15, four weeks after the development of an abscess, pneumothorax was started and six treatments were given, the date of the last being November 20, when the patient was discharged as cured. She remains well thirteen months after treatment.

CASE 3.-Mr. A. C. N., seen in consultation with Drs. D. E. Mulcahy and Reginald Walker, was subjected to a dotible frontal sinus operation, about Nov. 1, 1916, and developed an abscess of the right lung, November 7. Pneumothorax was started, November 24, and eight treatments were given, the date of the last being January 21 . The patient had a rather slow convalescence, but the lung was healed by March 1 , and he remains well, eleven months after treatment.

CASE 4.-Anna J., aged 13, seen in consultation with Dr. Claytor, was operated on, about October 15 , developing abscess of the left lung one week later. The patient became progressively worse, and pneumothorax was started, November 27. Five treatments were given, the last one, December 30, when the patient was discharged as cured. So far as I am able to learn, she remains well eleven months after treatment.

CASE 5.--Mrs. L. H., aged 24, seen in consultation with Dr. Charles White, was operated on, about Jan. 15, 1917, and developed an abscess of the right lung one week later. I was called in, February 22, one month after the development of the abscess. The patient gave a history of having suffered with asthma and epileptic attacks for a number of years. Examination revealed a probable five months' pregnancy, although there was no fetal motion or fetal heart sounds. The patient was desperately sick. Two treatments were given, producing a partial compression of the lung. The patient coughed up a large amount of pus and seemed somewhat better for tweny-four hours. She went into labor, however, March 1, and delivered a five months' fetus, which had apparently been dead several weeks. No more injections of gas were attempted. She died, March 4, two days after the delivery of the fetus. In this patient, the history of asthma and epilepsy showed a poor general condition, and the additional complication of the pregnancy with miscarriage made the case hopeless.

CASE 6.-Miss K. S., aged 24 , seen in consultation with Drs. C. W. Richardson and G. N. Acker, was operated on, March 15, 1917. An abscess in the right lung developed, March 20. The patient was very septic, becoming progressively worse. Pneumothorax was started, March 29, nine days after onset of the abscess. Seven treatments were given, the date of the last being May 7, when the patient was discharged as cured. She remains perfectly well at the present time, more than seven months after discharge.

CASE 7.--Miss K. K., aged 20, seen in consultation with Drs. C. B. Conklin and Charles White, was operated on, May 9, 1917, and developed an abscess of the right lung one week later. Pneumothorax was started, June 4, nineteen days after the development of the abscess. It was noticed at the first treatment that there was a little fluid in the pleural sac. Three injections of gas were given at intervals during the period from June 4 to June 10 . The lower portion of the lung collapsed, but there were adhesions over the upper half of the lung, which prevented collapse. The patient developed considerable pain, and the signs of pleural effusion increased rapidly. We suspected a rupture of the abscess into the pleural sac, and aspiration confirmed our suspicion. We found the pleural sac to contain a thin, slightly turbid fluid, of an extremely foul odor. The patient was immediately taken to the hospital, where Dr. Charles White did a partial rib resection and put in a drainage tube. The infection was extremely virulent, showing chiefly streptococci. The patient showed no improvement after the operation, and died, June 23. This case was a great disappointment, for while the patient was desperately ill before pneumothorax was begun, still her symptoms seemed no worse than those of several patients who were cured. The presence of pleural adhesions was probably the cause of the rupture of the abscess into the pleural sac. This fact impresses on us the importance of collapsing these cases promptly. The longer we wait, the more probable it is that we shall find pleural adhesions.

CASE 8.-Hazel H., aged 13, seen in consultation with $\mathrm{Dr}$. J. D. Rogers, was operated on early in June, and developed an abscess in the right lung about June 15 . The patient appeared very septic, and pneumothorax was started, June 28, two weeks after the development of the abscess. Five treatments were given during the period between June 28 and July 25 , when the patient was discharged as cured. She remains well five months after the discharge.

\section{The following are two cases of chronic lung abscess:}

CASE 9.-Mr. A. B., aged 25, was seen in consultation with Dr. Herbert Martin, Nov. 24, 1916. His tonsils had been removed one year before, an operation which had been followed by an acute abscess of the right lung. The abscess had apparently been healing gradually, and the patient thought himself nearly well. Early in November, 1916, however, he suddenly began to have a severe pulmonary hemorrhage. Dr. Martin had tried all the usual methods of treatment, but the patient continued to have a severe hemorrhage about every twenty-four hours. Examination revealed signs of an abscess involving the middle and the lower portions of the right lung. Pneumothorax was started, November 24, and was continued until January 24, eight treatments in all being given. It took about three weeks entirely to control the hemorrhage, and it was impossible to get a complete compression of the lung on account of the dense scar around the abscess in the lung tissue. The patient improved somewhat, and was up and around, but still had a slight elevation of temperature, and coughed up large amounts of pus. I understand that he has since had a radical operation performed, consisting of the removal of more than half the right lung, and that he has improved sufficiently to return to work. Pneumothorax probably prevented him from bleeding to death, but on account of the impossibility of our obtaining a complete compression of the lung, a cure could not be effected.

CASE 10.-Mrs. J. P., aged 29, was seen in consultation with Dr. Claytor. Her tonsils had been removed two years previously, the operation being followed by an acute abscess of the left lung. The abscess had become chronic and she had continued to cough up pus for a year. She had then been operated on by Dr. James Mitchell, who had resected one rib posteriorly, opened up the abscess and put in a drainage tube. Her general condition had improved, but she had still continued to drain and cough up pus. I started pneumothorax, April 25, 1917, and continued it until May 27, giving six treatments. On account of pleural adhesions and scars in the lung, only a partial collapse was obtained. For a few weeks the expectoration and drainage diminished very materially, but the improvement was only temporary. Her general condition remains good, but she will probably never be well unless she has a resection of the lung.

\section{SUMMARY AND CONCLUSIONS}

Of the ten cases of pulmonary abscess treated, six patients, or 60 per cent., were cured; two, or 20 per cent., were only temporarily improved, and two, or 20 per cent., died. Of the ten patients treated, seven 
had the abscess in the right lung, and three had the abscess in the left lung. In four cases in which the abscess was of less than two weeks' duration, a prompt cure was effected. In six cases in which the abscess was of longer duration, two patients were cured, two were temporarily improved, and two died.

In view of the poor results obtained by the usual procedures, pneumothorax used promptly appears to be the rational treatment in all cases of acute pulmonary abscess. In most cases of chronic pulmonary abscess the patients will probably not be materially benefited by the use of pneumothorax.

4107 Fourteenth Street N.W.

\section{HEMORRHAGE INTO AN OVARIAN CYST SIMULATING ECTOPIC PREG- NANCY *}

\section{CHESTER H. WATERS, M.D. OMAHA}

The attention of a small group of observers has been attracted to the clinical resemblance of certain ovarian conditions to ectopic pregnancy. The majority of cases recorded have dealt with the formation of a pelvic hematocele of ovarian origin, usually due to rupture and subsequent hemorrhage from a corpus luteum cyst.

The most important ovarian conditions that may suggest ectopic pregnancy are: (1) a corpus luteum or follicular cyst; (2) torsion of the pedicle of a cyst; (3) rupture of a cyst with or without intra-abdominal hemorrhage ; (4) ovarian hemorrhage, and (5) hemorrhage into a cyst. Judging from the literature, the last is infrequent, especially as regards its giving rise to symptoms suggestive of ectopic pregnancy.

The exceedingly close simulation of ectopic pregnancy is well shown in the following instance:

History-Mrs. D., aged 28, secundipara, whose labors had been normal, the last one occurring fourteen months before, consulted me, Aug. 27, 1916, fearing pregnancy. At that time menstruation was one week overdue. She was in good general health, and menstruation had been entirely regular and normal previously except for some slight discomfort until the flow was established. The cervix was of normal consistency, the fundus not enlarged; both ovaries were distinctly palpable, the left being slightly enlarged and tender. The adnexa, otherwise, were negative. Nothing, in short, besides the nonappearance of the menses was found to suggest pregnancy, and an effort was made to reassure the patient of its improbability. When called three days later, I found the patient suffering with severe abdominal pain, and flowing profusely. The husband, insufficiently reassured of the unlikelihood of pregnancy, had procured some "pills" from another physician to "bring her around." Several of these had been taken on the preceding day. During the night, colicky pains in the lower abdomen occurred, increasing in severity, and accompanied by diarrhea and a profuse vaginal flow. Large clots of blood and fragments of "tissue" were passed, all of which, fortunately, were saved and examined grossly and microscopically. The patient's temperature was 101, and the pulse rate 90 and of good quality. There were marked tenderness and rigidity on the left side of the lower abdomen, but no mass could be felt and no free fluid was demonstrable. Nausea was present, but no vomiting.

Bimanual examination revealed the same condition of the uterus and cervix as was found several days previously,

* From the surgical and pathological departments, University of Nebraska College of Medicine. except that the external os was slightly patulous. On the left side of the pelvis, however, a tender globular and cystic mass the size of a goose egg could be determined. It was but slightly movable, and corresponded in position to the ampullary region of the tube or the ovary. There was no bulging of the culdesac. As the general condition, quality and rate of the pulse did not suggest intra-abdominal hemorrhage, an expectant procedure was adopted with absolute rest in bed and application of an ice bag over the lower abdomen.

The tissue passed by the vagina was found to consist of practically a complete triangular cast of the uterine cavity, such as will often be passed in cases of ectopic gestation after intra-abdominal rupture or operative removal. Microscopically, the cast was shown to be a rather hyperplastic endometrium, but it showed no decidual reaction or the presence of decidual cells. There was no evidence of chorionic villi or other fetal elements in either the membranes or the clots. Accordingly, the possibility of an intra-uterine pregnancy seemed to be eliminated and the likelihood of an ectopic gestation somewhat in doubt, for the endometrial casts in the latter condition usually show a decidual reaction.

On the following day the patjent's condition was about the same with regard to pain in the left side, though perhaps it was more constant and less colicky in nature. The flow had decreased somewhat. A leukocyte count on this day (September 2) showed a total white blood count of 18,000 , with polymorphonuclears 82 per cent. The temperature was 99.4. The pelvic condition was the same as on the preceding day, and the general condition, color and pulse manifested no evidence of internal hemorrhage.

During the next week in watchful expectancy the pain became less severe, though persistent, and the flow practically ceased. The temperature varied from normal to 100 , while the pulse rate rarely exceeded 90 . Loss of appetite and occasional nausea persisted. There was no demonstrable change in the pelvic condition noted previously. As there was no appreciable improvement in the local condition, operation was decided on. The diagnosis at the time lay between (1) hemorrhage into a corpus luteum; (2) a twist of the pedicle of an ovarian cyst; (3) tubal pregnancy unruptured, or (4) a tubal abortion. The clinical course and findings on examination favored the diagnosis of hemorrhage into a corpus luteum.

Operation and Result.-Sept. 11, 1916, a transverse incision revealed no free blood in the peritoneal cavity. The uterus was not enlarged; it was in midposition. The left ovary was transformed into a dark, tense cyst the size of a small apple. There was no torsion of its pedicle. The right ovary contained a corpus luteum of normal appearance. Both tubes were normal. Left ovariectomy, the Willis operation for shortening the round ligaments, and appendectomy were followed by dilatation of the cervix and curettage. The curettings were moderately abundant and showed, microscopically, complete regeneration of the endometrium with slight glandular hyperplasia.

Convalescence was uneventful, and menstruation since has been entirely painless and otherwise normal. Grossly, the left ovary was practically replaced by a tense, dark, thinwalled, unilocular cyst with dark bloody content. Microscopically, the cyst wall was shown to consist of fibrous and scanty stroma tissue without definitely distinguishable epithelial lining. No lutein cells were in evidence. In the basal portion of the cyst wall, where more ovarian tissue was present, there were numerous small areas of petechial or interstitial hemorrhage.

\section{COMMENT}

A retrospective analysis of this case presents several features of speculative interest:

1. The Causal Relationship of Ovarian Cysts to Perverted Menstruation.-The inhibitory influence of ovarian cysts, either of follicular or corpus luteum origin, on the periodicity of menstruation has been observed by Halban ${ }^{1}$ and more recently corroborated and substantiated by Rubin. ${ }^{2}$

1. Halban: Jour. Gynec., 1915, 34.

2. Rubin: Surg., Gynec.' and Öbst., 1917, 24, No. 4. 